\title{
Quarkonium binding and entropic force
}

\author{
Helmut Satz ${ }^{\mathrm{a}}$ \\ Fakultät für Physik, Universität Bielefeld, 33501 Bielefeld, Germany
}

Received: 2 February 2015 / Accepted: 20 April 2015 / Published online: 6 May 2015

(C) The Author(s) 2015. This article is published with open access at Springerlink.com

\begin{abstract}
A $Q \bar{Q}$ bound state represents a balance between repulsive kinetic and attractive potential energy. In a hot quark-gluon plasma, the interaction potential experiences medium effects. Color screening modifies the attractive binding force between the quarks, while the increase of entropy with $Q \bar{Q}$ separation gives rise to a growing repulsion. We study the role of these phenomena for in-medium $Q \bar{Q}$ binding and dissociation. It is found that the relevant potential for $Q \bar{Q}$ binding is the free energy $F$; with increasing $Q \bar{Q}$ separation, further binding through the internal energy $U$ is compensated by repulsive entropic effects.
\end{abstract}

\section{Introduction}

The concept of entropic forces, emerging as a result of collective many-body phenomena, has in recent times attracted increasing interest; see e.g. [1-4]. The effect of such forces arises from the thermodynamic drive of a many-body system to increase its entropy, rather than from a specific underlying microscopic force. This can also provide a way of studying the role of entropy maximization for a specific dynamic system immersed in a thermal medium. We here want to use this approach to address quarkonium binding and dissociation in a hot deconfined quark-gluon plasma [5].

The simplest approach to study $Q \bar{Q}$ binding in a medium of temperature $T$ is in terms of the Schrödinger equation

$$
\left[2 m_{Q}-\frac{1}{m_{Q}} \nabla^{2}+V(r, T)\right] \Phi_{i}(r, T)=M_{i}(T) \Phi_{i}(r, T) .
$$

Its solution gives the resulting quarkonium masses $M_{i}(T)$, with $i=0$ for the ground state and $i=1,2, \ldots$ for the subsequent excited states. Here $m_{Q}$ denotes the $c$ or $b$ quark mass, while $V(r, T)$ describes the in-medium binding potential. To obtain a feeling for the resulting behavior, it is helpful

\footnotetext{
a e-mail: satz@physik.uni-bielefeld.de
}

to consider the semi-classical limit of Eq. (1) [6],

$$
\left[2 m_{Q}+\frac{c}{m_{Q} r^{2}}+V(r, T)\right]=E(r, T),
$$

with $c$ a parameter of order unity, to be determined such as to give correct quarkonium masses. In vacuum, for $T=0$, the binding potential is generally assumed to have the Cornell form

$V(r, T=0)=\sigma r-\frac{\alpha}{r}$,

where $\sigma$ denotes the string tension and $\alpha$ the Coulombic running coupling. For illustration, we concentrate for the moment on the strong coupling form and neglect the Coulombic term; later on, we shall include it.

In vacuum, we thus set $V(r, T=0)=\sigma r$ and minimize the energy $E(r, T=0)$ with respect to $r$. This yields

$r_{0}=\left(\frac{2 c}{m_{Q} \sigma}\right)^{1 / 3}$

for the vacuum ground state $Q \bar{Q}$ separation and

$M_{0}(Q \bar{Q})=2 m_{Q}+\frac{3}{2}\left(\frac{2 c \sigma^{2}}{m_{Q}}\right)^{1 / 3}$

for the corresponding vacuum ground state mass. The behavior of $\left[E(r, 0)-2 m_{Q}\right]$ is illustrated in Fig. 1; the minimum arises from the competition between the kinetic energy term $c / m r^{2}$ and the binding potential $\sigma r$. With $\sigma=0.2 \mathrm{GeV}^{2}$, $m_{c}=1.3 \mathrm{GeV}$ and $c=1.3$, this yields $r_{0}(c \bar{c})=0.43 \mathrm{fm}$ for the diameter and $M_{0}(c \bar{c})=3.2 \mathrm{Gev}$ for the mass of the charmonium ground state. Similarly, the bottomonium sector gives with $m_{b}=4.6 \mathrm{GeV}$ and $c=1.1$ the values $r_{0}(b \bar{b})=0.27 \mathrm{fm}$ and $M(b \bar{b})=9.6 \mathrm{GeV}$. The bound states thus arise for parameter values for which the competition comes to a draw.

We now place the given $Q \bar{Q}$ pair into a deconfined medium of temperature $T$. This will have two distinct effects: the constituents of the medium will modify the interaction between the static quarks, and they will interact with each of 


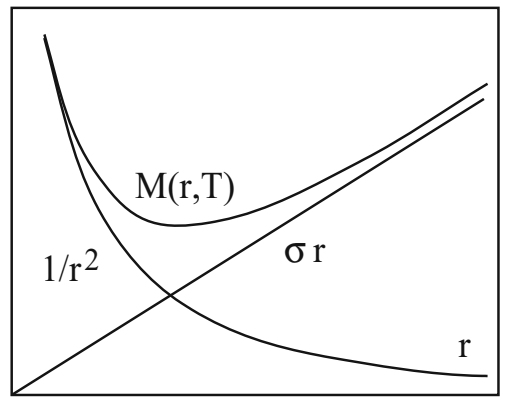

Fig. 1 Semi-classical form of quarkonium binding in vacuum

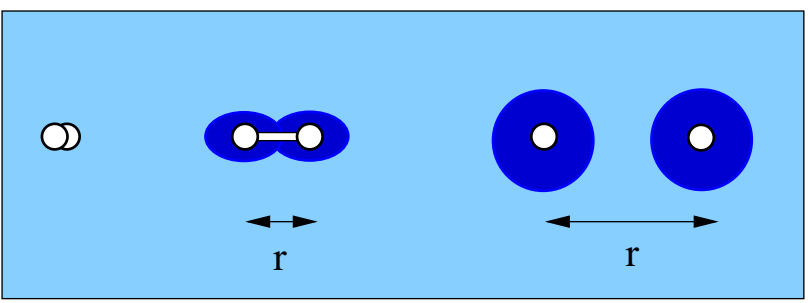

Fig. $2 Q \bar{Q}$ pair separation and polarization cloud formation

the static quarks individually. The binding of the pair is mediated by an exchange of virtual gluons, and this exchange is modified by their interaction with the on-shell constituents of the medium. As a result, the binding force itself is modified. On the other hand, the on-shell constituents of the medium can also interact directly and individually with the $Q$ and the $\bar{Q}$. For sufficiently large separation distance, this leads to the formation of polarization clouds around each heavy quark. On one hand, this requires an energy input to provide the increase of their effective mass; on the other hand, the entropy of the overall system grows. This entropy increases over a range of $r$, and the resulting entropic force acts to pull $Q$ and $\bar{Q}$ apart. Let us look at these possible modifications, starting with a static $Q \bar{Q}$ pair: in other words, we first consider the effect on the potential term only.

In the limit of small $r$, the two heavy quarks form a color neutral entity, so the medium does not see them, nor do they see the medium. The only energy difference between our medium and one without a $Q \bar{Q}$ pair is thus the mass of the small $Q \bar{Q}$ system. To further separate $Q$ and $\bar{Q}$, work has to be done against the interquark binding force. Increasing the temperature of the medium will modify the binding, and this of course has to be taken into account. Once the quarks are sufficiently separated, their color charges begin to induce polarization effects in the medium, first forming a cloud around the pair. With further separation, the quarks interact less strongly with each other, but the medium now forms clouds around the individual $Q$ and $\bar{Q}$. The overall picture is schematically illustrated in Fig. 2.

For a small imagined volume containing the $Q \bar{Q}$ pair, we thus note two consequences. For a separation increase $\Delta r$, the string energy grows by $\sigma \Delta r$; in addition, the effective mass of each heavy quark begins to increase, due to the onset of polarization. This means that the overall energy in the volume increases. On the other hand, the rearrangement of the constituents of the medium to form polarization clouds around each heavy quark results as well in an increase of the relevant entropy. In view of this interplay of energetic and entropic effects, the form of the potential to be used in the Schrödinger equation (1) has been the subject of considerable discussion. Proposals range from the free energy, $V(T, r)=F(T, r)$, to the internal energy, $V(T, r)=U(T, r)$, and include even arbitrary combinations of the two [7-13]. We want to show here that a careful study of the underlying forces can resolve this issue to some extent.

In the next section, we shall first address the behavior of the system in the strong coupling regime, where the thermodynamics can be formulated in terms of a remnant string tension as binding. Following that, we turn to the weak coupling regime, in which the interquark forces are of Coulombic nature. In a final section, we shall then address the specific effects arising in the region dominated by critical behavior.

\section{The strong coupling regime}

To study this region in more detail, we consider a specific simple model, based on a remnant string tension $\sigma$ as binding force in a deconfined plasma. To begin, we assume the medium to give rise to a screening mass $\mu(T)=$ const. $T$; the modification of $\mu(T)$ by critical behavior near the deconfinement point $T_{c}$ will be addressed later on. For the free energy of the pair in the medium we assume the form [6]

$F(r, T)=\sigma r\left[\frac{1-\mathrm{e}^{-\mu r}}{\mu r}\right]=\frac{\sigma}{\mu}\left[1-\mathrm{e}^{-x}\right], \quad x=\mu r$,

with a screening factor $\left(1-\mathrm{e}^{-\mu r}\right) / \mu r$ based on onedimensional QED [14,15]. We have here neglected short distance Coulombic effects on the quark binding; we shall also return to these later. The expression (6) specifies the free energy difference between a plasma containing a $Q \bar{Q}$ pair and one at the same temperature without such a pair. All further thermodynamic quantities will also describe the corresponding differences; we will not explicitly note that in each case in the following.

The corresponding entropy becomes

$T S(T, r)=-T\left(\frac{\partial F}{\partial T}\right)_{r}=\left(\frac{\sigma}{\mu}\right)\left[1-(1+x) \mathrm{e}^{-x}\right]$,

with the assumed screening mass linear in $T$, so that $T(\mathrm{~d} \mu / \mathrm{d} T)=\mu$. We note that in the short distance limit, the entropy vanishes, as expected. In the large distance limit at constant temperature, on the other hand, it attains a constant 


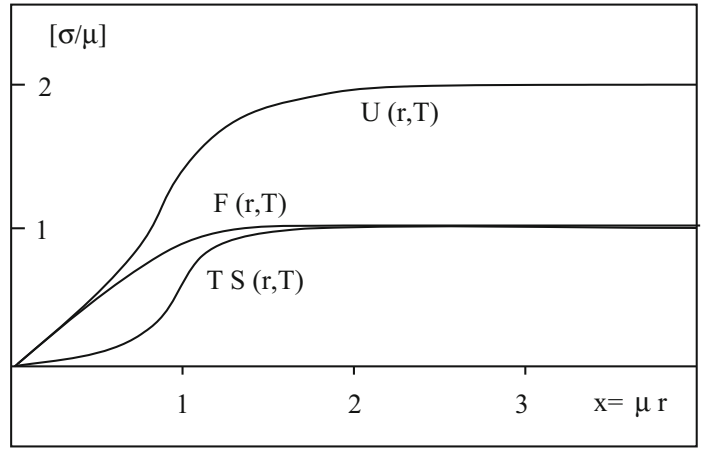

Fig. 3 Strong coupling form of thermodynamic $Q \bar{Q}$ potentials

value, corresponding to the entropy of the two polarization clouds.

From the free energy and the entropy, we obtain the total (internal) energy difference for the system,

$U(T, r)=F(T, r)+T S(T, r)=\left(\frac{\sigma}{\mu}\right)\left[2-(2+x) \mathrm{e}^{-x}\right]$,

consisting of one term accounting for the work done against the string tension and one for the formation of the polarization clouds. In the present model, each of the terms contributes in the large distance limit an equal amount $\sigma / \mu$ to the total energy. In Fig. 3 the resulting behavior of free energy, entropy, and total energy are illustrated. The total energy initially increases because the free energy does, but for $x \gtrsim 1$, it does so because of the formation of the polarization masses.

The resulting pressure $P(T, r)$ is given by

$P(T, r)=-\left(\frac{\partial F}{\partial r}\right)_{T}=-\sigma \mathrm{e}^{-x}$

It is negative, indicating that the $Q \bar{Q}$ binding is attractive. Its absolute value decreases with increasing $\mu$, indicating that growing temperature enhances screening and hence weakens the binding, causing it to vanish in the high temperature limit. Similarly, at fixed $\mu$, color screening strongly reduces the binding for separation distances $r>r_{D}=1 / \mu$, when quark and antiquark can no longer communicate.

The pressure specifies the force acting on the $Q \bar{Q}$ pair. It consists of two distinct terms; from the relation $F=U-T S$ between free energy, internal energy, and entropy we have

$$
\begin{aligned}
P(T, r) & =-\left(\frac{\partial U}{\partial r}\right)_{T}+T\left(\frac{\partial S}{\partial r}\right)_{T} \\
& =K_{u}(T, r)+K_{s}(T, r) .
\end{aligned}
$$

Here $K_{u}=-(\partial U / \partial r)_{T}$ denotes the energetic force and $K_{S}=(\partial S / \partial r)_{T}$ the entropic force. This specific form of the force is known from the thermodynamics of a gas retained by a moving piston. The volume there becomes $\mathrm{d} V=A \mathrm{~d} r$, with $A$ for the cross-section area of the piston. Since the force is the pressure per area, $K=P A$, the relation $P=T(\partial S / \partial V)=(T / A)(\partial S / \partial r)$, and similarly for $U$, then leads to the form of the forces employed in Eq. (10).

Both $(\partial U / \partial r)_{T}$ and $(\partial S / \partial r)_{T}$ increase for increasing $r$. The former increases first by the energy spent to separate the pair against the attractive string tension and then by the energy needed to form the polarization clouds acquired by the separated quarks. The latter does so because the increase in the constituent density of the medium around $Q$ and $\bar{Q}$ increases the overall entropy. The two forces $K_{u}$ and $K_{s}$ thus act in opposite directions, with $K_{u}$ attractive and $K_{s}$ repulsive; hence they largely cancel each other. For the above model, we obtain

$P(T, r)=\left[-\sigma(1+x) \mathrm{e}^{-x}\right]+\left[\sigma x \mathrm{e}^{-x}\right]=-\sigma \mathrm{e}^{-x}$,

with the two terms in square brackets denoting energetic and entropic force, respectively. Their behavior as a function of $r$ at fixed temperature is illustrated in Fig. 4. At short distances, the energetic force dominates, while in the large distance limit, the ratio of the two forces approaches unity.

The overall force acting on the $Q \bar{Q}$ pair is given by the pressure; since it is obtained from the free energy, $F(T, r)$ is the relevant binding potential. The steeper potential increase and hence stronger binding obtained when $U(T, r)$ is used
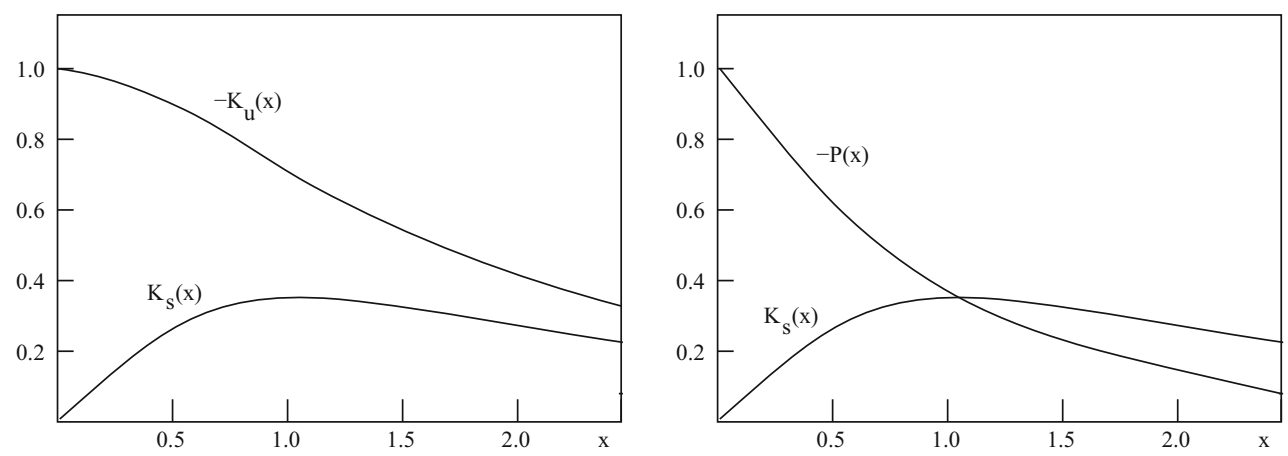

Fig. 4 Strong coupling forms of energetic and entropic forces and of pressure, in units of $\sigma$ 
in the Schrödinger equation [7-9, 11] arise only because the repulsive entropic force is ignored; it in fact compensates the stronger attraction suggested by $U$. The overall effect on the $Q \bar{Q}$ is due to a combination of the two opposing forces, and this leads to $F(T, r)$. Nevertheless, one may still question the validity of the two-body Schrödinger approach in regions in which the repulsive entropic force is significantly larger than overall pressure, i.e., for $x \gtrsim 1$. In this region, the combined strength of the interaction between the $Q$ and the on-shell constituents of the medium is stronger than that with the $\bar{Q}$.

Before turning to the weak coupling regime, we note that through the Maxwell relation

$T\left(\frac{\partial P}{\partial T}\right)_{r}=T\left(\frac{\partial S}{\partial r}\right)_{T}=K_{s}(T, r)=\sigma x \mathrm{e}^{-x}$

the temperature dependence of the pressure at fixed $r$ is in fact determined by the entropic force alone.

\section{The weak coupling regime}

Here the interquark potential is of screened $1 / r$ type, so that the difference in free energy between a system with and one without a $Q \bar{Q}$ pair is given by

$F(T, r)=-\alpha \frac{\mathrm{e}^{-\mu r}}{r}-\alpha \mu$

again $\mu(T) \sim T$ is the color screening length in the medium, $r_{D}=1 / \mu$ the Debye radius. The second term above accounts for the effect of the polarization clouds at infinite separation, and $\alpha$ denotes the (running) Coulombic coupling. Defining $x=\mu r$, we rewrite Eq. (13) as

$F(T, r)=-\alpha \mu\left[1+\frac{\mathrm{e}^{-x}}{x}\right]$

The corresponding entropy difference is given by

$T S(T, r)=-T\left(\frac{\partial F}{\partial T}\right)_{r}=\alpha \mu\left[1-\mathrm{e}^{-x}\right]$

it vanishes for $r=0$ and also approaches the individual cloud contributions in the large distance limit. From free energy and entropy we obtain the total energy difference

$U(T, r)=F(T, r)+T S(T, r)=-\alpha \mu\left[1+\frac{1}{x}\right] \mathrm{e}^{-x}$.

It is seen to approach the free energy form for small $r$, while vanishing in the large distance limit, where free energy and entropy cancel each other: the work done in separating $Q$ and $\bar{Q}$ is balanced by the increase of entropy. The behavior of the three thermodynamic potentials is illustrated in Fig. 5.

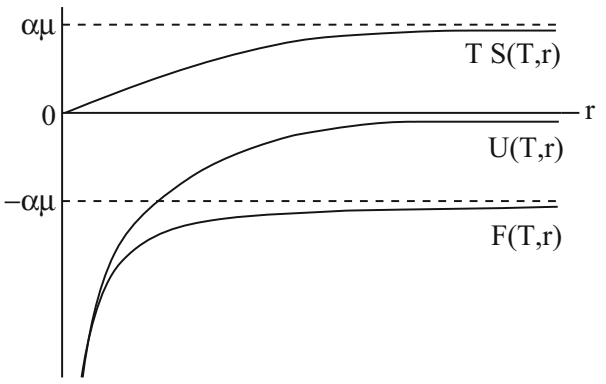

Fig. 5 Weak coupling form of thermodynamic $Q \bar{Q}$ potentials

From the free energy, we obtain for the resulting pressure

$$
\begin{aligned}
P(T, r) & =-\left(\frac{\partial F}{\partial r}\right)_{T}=-\alpha \mu\left[\frac{1}{\mu r}+1\right] \frac{\mathrm{e}^{-\mu r}}{r} \\
& =-\alpha \mu^{2}\left[\frac{1}{x^{2}}+\frac{1}{x}\right] \mathrm{e}^{-x} .
\end{aligned}
$$

It is negative, indicating that also here there is a force acting to contract the pair. Its absolute value decreases with increasing separation distance $r$ and vanishes in the large distance limit.

The pressure again consists of an entropic and an energetic force. The former is given by

$K_{S}(T, r)=T\left(\frac{\partial S}{\partial r}\right)_{T}=\alpha \mu^{2} \mathrm{e}^{-x}$.

It is positive, enhancing the dissociation of the pair. The corresponding energetic force becomes

$K_{u}(T, r)=-\left(\frac{\partial U}{\partial r}\right)_{T}=-\alpha \mu^{2}\left[\frac{1}{x^{2}}+\frac{1}{x}+1\right] \mathrm{e}^{-x}$

It dominates in the short distance limit, while for large distances the entropic and energetic forces become equal and cancel each other, resulting in a vanishing overall pressure. Through the Maxwell relation

$T\left(\frac{\partial P}{\partial T}\right)_{r}=T\left(\frac{\partial S}{\partial r}\right)_{T}=K_{S}(T, r)=\alpha \mu^{2} \mathrm{e}^{-x}$

the entropic force again determines the temperature variation of the pressure.

\section{The critical regime}

So far, we had assumed the screening mass to increase linearly in $T$. This assumption evidently becomes incorrect when the temperature drops to the region just above the deconfinement point. The behavior of $\mu(T)$ is illustrated schematically in Fig. 6; approaching $T_{c}, \mu(T)$ abruptly drops to a small value determined by string breaking in the confined phase. This pattern affects in particular the thermodynamic potentials involving $\mathrm{d} \mu / \mathrm{d} T$, which increases very sharply near $T_{c}$ and in principle could even diverge there. 


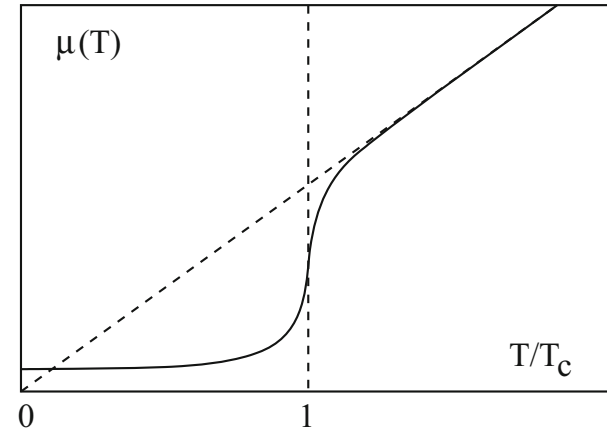

Fig. 6 The temperature variation of the screening mass $\mu(T)$

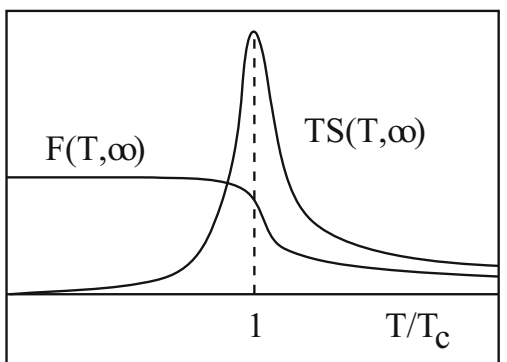

Fig. 7 Large distance limits of free energy and entropy vs. temperature

For $\mu(T)$ decreasing to very small values, the free energy

$F(r, T)=\frac{\sigma}{\mu}\left[1-\mathrm{e}^{-x}\right]-\alpha \mu\left[1+\frac{\mathrm{e}^{-x}}{x}\right]$

essentially falls back to the Cornell potential

$F(T, r) \simeq \sigma r-\frac{\alpha}{r}$.

Here and in the following, we shall consider the full thermodynamic potentials, combining the expression for the strong and weak coupling limits. Corresponding to Eq. (21), the pressure becomes

$P(T, r)=-\left[\sigma+\alpha \mu^{2}\left(\frac{1}{x}+\frac{1}{x^{2}}\right)\right] \mathrm{e}^{-x}$
In the small distance limit, it also reduces to the attractive force in vacuum,

$P(T, r) \simeq-\left(\sigma+\frac{\alpha}{r^{2}}\right)$.

In both cases, medium effects become significant only at large $r$, where the screening factor $\exp \{-\mu r\}$ becomes significantly less than unity and begins to play a role.

In contrast to free energy and pressure, the entropy, and the corresponding force contain temperature derivatives of the screening mass, $\mathrm{d} \mu / \mathrm{d} T$, which near the critical deconfinement temperature increase very sharply. The entropy is given by

$S(T, r)=(\mathrm{d} \mu / \mathrm{d} T)\left(\frac{\sigma}{\mu^{2}}\left[1-(1+x) \mathrm{e}^{-x}\right]+\alpha\left[1-\mathrm{e}^{-x}\right]\right)$,

and the entropic force becomes

$K_{S}(T, r)=T(\mathrm{~d} \mu / \mathrm{d} T)\left(\frac{\sigma}{\mu} x \mathrm{e}^{-x}+\alpha \mu \mathrm{e}^{-x}\right)$.

For completeness, we note that

$$
\begin{aligned}
U(T, r)= & T(\mathrm{~d} \mu / \mathrm{d} T)\left(\frac{\sigma}{\mu^{2}}\right) \\
& \times\left\{\left[2-(2+x) \mathrm{e}^{-x}\right]-\frac{\alpha}{\mu}\left[1+\frac{1}{x}\right] \mathrm{e}^{-x}\right\}
\end{aligned}
$$

gives the total internal energy of the system. It again contains the temperature derivative of the screening mass and thus strongly reflects the critical behavior near $T_{c}$.

To obtain a first idea of the role of critical behavior, we compare in Fig. 7 the large distance forms of free energy and entropy. It is evident that near the critical point, there is a sudden increase of the entropy, caused by the increase or divergence of the correlation length, which now connects ever larger regions of the medium.

To study the actual behavior of the relevant forces, we turn to finite temperature lattice studies [16-18]. In Fig. 8 we show results for two different temperatures, $T=190$ and $T=270 \mathrm{MeV}$. The lower temperature is quite close to the deconfinement value of about $160 \mathrm{MeV}$. It is seen that
Fig. 8 The pressure $P(r, T)$ (blue) and the entropic force $K_{S}(r, T)(r e d)$ as a function of $r$ for two temperature values [16-19]; the forces are given in units of $\left(\mathrm{fm}^{-2}\right)$
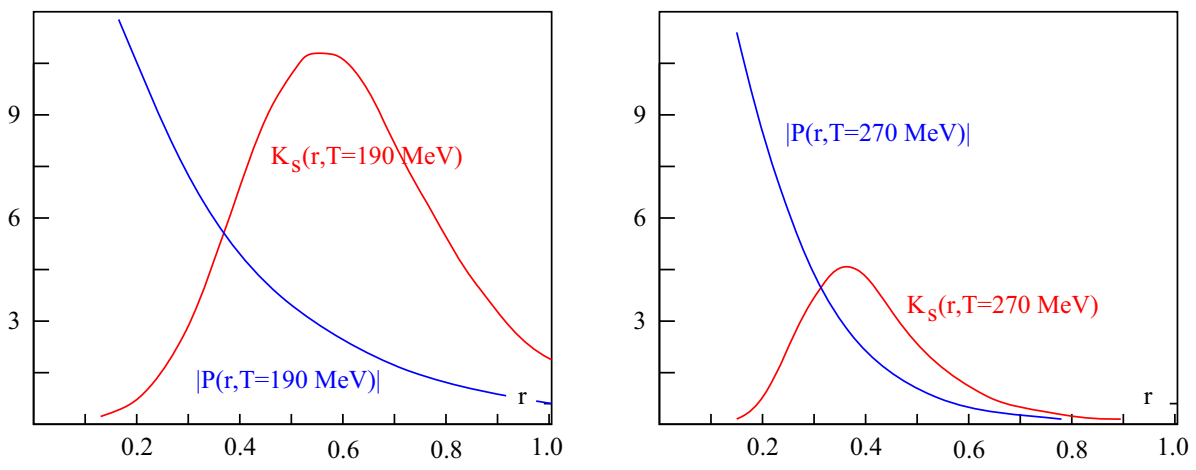
here the entropic force becomes strongly enhanced relative to the pressure, and in range of distances around $0.5 \mathrm{fm}$ it is in fact much larger. The low pressure values thus reflect the cancelation of entropic and energetic forces in this region.

The striking and perhaps surprising conclusion thus is that the divergent critical effects near $T_{c}$ in fact cancel out for the $Q \bar{Q}$ binding; the overall potential between $Q$ and $\bar{Q}$ only notices a sudden decrease at the critical point. Here the (negative) pressure is simply reduced considerably, allowing an easier dissociation than in vacuum. In a potential approach based on equilibrium thermodynamics and the two-body Schrödinger equation (1), there is thus no special entropic critical effect causing stronger quarkonium dissociation.

Nevertheless, it is evident from Figs. 7 and 8 that both entropy and entropic force show sharp increases near $T_{c}$. This indicates that in the relevant $r$ regions, the interaction between a heavy quark and the combined constituents of the medium becomes much stronger than that between $Q$ and $\bar{Q}$. This may well be a signal that here a two-body potential approach is no longer applicable, allowing an enhanced collective entropic quarkonium dissociation [4].

\section{Concluding remarks}

In summary, we conclude that if quarkonium binding and dissociation is to be studied in terms of a potential approach, the relevant potential is the free energy difference $F(T, r)$ between a medium with and one without a $Q \bar{Q}$ pair. The stronger increase of the internal energy $U(T, r)$ with increasing separation distance $r$ is compensated by equally strong contributions from the repulsive entropic force, opposing rather than enhancing any binding. Hence $U$ as a potential only makes sense when these are not present, i.e., when $U \simeq F$

The conclusion that the free energy is the appropriate potential for $Q$ binding is also in accord with previous work comparing real and imaginary time $Q \bar{Q}$ correlators in a thermal medium [20]. Very recently, it was obtained as well in a potential derivation from real-time Wilson loop studies [21].

At first sight, the long-standing question, $F$ or $U$ ?, thus appears to be resolved in favor of $F$. However, as long as the role of larger distance repulsive entropic effects is not fully clarified, it remains conceivable that a potential approach as such only makes sense in that $(T, r)$ region where $F \simeq U$. This is the point of view adopted in [4], where the strong increase of the entropy and the ensuing entropic force in the temperature region around $T_{C}$ are taken to rule out a description in terms of a binding potential when $F \neq U$. Our present work is not meant to resolve this issue; it only gives the relevant form to be used in the case such a description is employed. Finally, we note that we have here addressed only the real part of the potential. In more formal studies [22], the important issue of its imaginary part, reflecting Landau damping and singlet-octet transitions, can be addressed as well. This aspect is beyond the scope of the present work; for recent studies and further literature, see [20-24].

Acknowledgments The paper of D. Kharzeev [4] was a crucial stimulus for this work, even though its topical focus differs from ours. It is a pleasure to thank him for sending it to me prior to publication and for several stimulating and helpful exchanges. I also want to thank F. Karsch for critical readings of earlier drafts of this work and providing most helpful input. Further thanks go to O. Kaczmarek and P. Petreczky for stimulating discussions, and to N. Brambilla and A. Vairo for helpful comments. The hospitality of the Institute for Nuclear Theory, University of Washington, and the partial support of the Department of Energy during the start of this work are gratefully acknowledged.

Open Access This article is distributed under the terms of the Creative Commons Attribution 4.0 International License (http://creativecomm ons.org/licenses/by/4.0/), which permits unrestricted use, distribution, and reproduction in any medium, provided you give appropriate credit to the original author(s) and the source, provide a link to the Creative Commons license, and indicate if changes were made. Funded by $\mathrm{SCOAP}^{3}$.

\section{References}

1. R.M. Neumann, Am. J. Phys. 48, 354 (1980)

2. E.P. Verlinde, JHEP 1104, 029 (2011)

3. P.G.O. Freund, arXiv: 1008.4147

4. D.E. Kharzeev, Phys. Rev. D 90, 074007 (2014)

5. T. Matsui, H. Satz, Phys. Lett. B 178, 416 (1986)

6. F. Karsch, M.-T. Mehr, H. Satz, Z. Phys, C 37, 617 (1988)

7. S. Digal, P. Petreczky, H. Satz, Phys. Lett. B 514, 57 (2001)

8. E. Shuryak, I. Zahed, Phys. Rev. D 70, 054507 (2004)

9. C.-Y. Wong, Phys. Rev. C 72, 034906 (2004)

10. C.-Y. Wong, Phys. Rev. C 76, 014902 (2007)

11. W. Alberico et al., Phys. Rev. D 72, 114011 (2005)

12. W. Alberico et al., Phys. Rev. D 75, 074009 (2007)

13. H. Satz, Extreme states of matter in strong interaction physics. in Lecture Notes in Physics, vol. 841 (Springer, New York, 2012) (chapter 8)

14. V.V. Dixit, Mod. Phys. Lett. A 5, 227 (1990)

15. S. Digal, P. Petreczky, H. Satz, Eur. Phys. J. C 43, 71 (2005)

16. O. Kaczmarek, F. Zantow, PR D 71, 114510 (2005)

17. O. Kaczmarek, F. Zantow, PoS LAT 2005, 177 (2006)

18. O. Kaczmarek, F. Zantow, arXiv:0710.0498

19. H. Satz, J. Phys. G36, 064011 (2009)

20. A. Beraudo, J.-P. Blaizot, C. Ratti, Nucl. Phys. A 806, 312 (2008)

21. Y. Burnier, O. Kaczmarek, A. Rothkopf, arXiv:1410.7311, arXiv: 1411.3141

22. N. Brambilla et al., arXiv:0804.0993

23. M. Laine et al., JHEP 0703, 054 (2007)

24. M. Laine, O. Philipsen, M. Tassler, JHEP 0709, 066 (2007) 